\title{
Very Long Chain Lipids Favor the Formation of a Homogeneous Phase in Stratum Corneum Model Membranes
}

\author{
Adrian Paz Ramos, Joke A. Bouwstra, and Michel Lafleur*
}

Cite This: Langmuir 2020, 36, 13899-13907

Read Online

ABSTRACT: The stratum corneum (SC), the outermost layer of mammal epidermis, acts as a barrier dictating the rate of absorption of exogenous molecules through the skin, as well as to prevent excessive water loss from the body. The SC consists of protein-rich corneocytes embedded into a complex lipid mixture. The lipid fraction is mainly constituted of an equimolar mixture of ceramides (Cer), free fatty acids (FFA), and cholesterol (Chol), forming a solid phase in the intracellular space; this lipid phase is supposed to play a fundamental role in the SC barrier function. An unusual characteristic of this biological membrane is that its lipids generally bear very long acyl chains, with the 24-carbon long ones being the most abundant. In this work, we used Raman microspectroscopy and infrared spectroscopy to study the influence of the acyl chain length on the lipid mixing properties in SC model membranes. Our results revealed that the combination of ceramides and FFA bearing a very long chain is required for the formation of homogeneous lipid mixtures, while lipids with shorter chains (16-carbon and 20-carbon atom long) lead to domains with micrometer dimensions. It is proposed that the biological machinery necessary for acyl chain elongation occurring at the mammalian skin level is required to inhibit lipid phase separation, a critical feature in the proper barrier functioning.

\section{INTRODUCTION}

The stratum corneum (SC) is the outermost layer of mammal epidermis and the primary diffusion barrier for penetrating substances and water loss across the skin. The SC is mainly composed of flattened and fully keratinized dead cells (corneocytes), embedded in a lipid matrix, which is mostly constituted of ceramides (Cer), free fatty acids (FFA), and cholesterol (Chol). ${ }^{1}$ The diffusion of molecules across SC is essentially intercellular as partitioning into corneocytes is severely limited and the lipid matrix forms a continuous element. ${ }^{2}$ Therefore, detailed knowledge about the structural organization of lipids in the SC is essential to rationalize and control skin impermeability.

SC lipid organization is complex. Several experimental evidences establish the coexistence of long- and short-repeat distance structures. ${ }^{3,4}$ The coexistence of orthorhombic and hexagonal lateral organization has also been shown., ${ }^{5,6}$ The different phases show a SC-depth dependence ${ }^{5,6}$ and may be associated with the presence of specific lipids (e.g., ceramide EOS is proposed to be essential for the formation of a longrepeat distance structure) ${ }^{7,8}$ Beyond the coexistence of these phases whose overall organization is not firmly established, extensive lipid phase separations would be a structural feature that should impact the skin impermeability as grain boundaries usually facilitate the diffusion process. ${ }^{9,10}$ It is rather challenging to assess in situ the spatial lipid distribution in the SC lipid phase because the lipid phase represents only $\sim 10$ $(\mathrm{w} / \mathrm{w}) \%$ of the SC and the thickness of lipid intercorneocyte spacing is very limited, under $100 \mathrm{~nm}^{2,11}$ Therefore, almost all the current knowledge relative to the mixing properties of SC lipids has been derived from model mixtures. It is established, from studies characterizing the behavior of model SC lipid mixtures, that SC lipid mixing properties depend on several factors.

Differences in the molecular architecture and variations in chain length distribution affect lipid miscibility. ${ }^{12-16}$ Brief et al. ${ }^{14}$ showed that the miscibility of CerNS16, a ceramide bearing a sphingosine base ( $S$ ) and an amide-linked nonhydroxylated (N) C16 chain, with palmitic acid (FFA16) and cholesterol was limited despite the fact that the three lipid species possess an optimized hydrophobic matching; this finding highlights the significance of the interactions at the head group level. Oguri et al. ${ }^{13}$ showed the impact of the FFA chain length on the mixing properties of SC model membranes constituted of a ceramide bearing a non-hydroxylated C24 chain (CerNS24), FFA, and cholesterol. They observed a phase separation when using FFA16, whereas mixtures with the very long lignoceric acid (FFA24) formed more homogeneous solid phases.

The lipid mixing properties are also influenced by the length of the non-hydroxy fatty acid of ceramides. Školová et al. ${ }^{15}$ and Pullmannová et al. ${ }^{16}$ showed that mixtures with cholesterol,

Received: August 5, 2020

Revised: September 28, 2020

Published: November 10, 2020 
FFA24, and CerNS bearing a long C16 chain were mostly phase-separated, whereas CerNS with a very long C24 acyl chain mixed well with the other components. Mixtures of bovine brain ceramides (BBCer), which bear mainly stearoyl (saturated C18) and nervonoyl (C24 with a cis unsaturation at positions 9-10) as the non-hydroxy amide-linked chain, cholesterol, and a single FFA with a chain length varying between $\mathrm{C} 14$ and $\mathrm{C} 22$, all showed limited miscibility; these model mixtures formed coexisting solid domains rich in either BBCer or FFA. ${ }^{12}$

In addition to the chemical features, domain formation is modulated by the thermal history of the samples as well as the kinetics. For example, a ternary mixture containing CerNS16, FFA16, and cholesterol that was quenched from a high temperature formed phase-separated solid phases after more than 90 days of incubation at $5{ }^{\circ} \mathrm{C}$, while crystalline domains were obtained after only $10 \mathrm{~min}$ at $40{ }^{\circ} \mathrm{C} .{ }^{17}$ Similarly, solid FFA16 and solid cholesterol domains were reported to form as a function of time in a BBCer/FFA16/Chol mixture; ${ }^{18}$ it took $\sim 55 \mathrm{~h}$ for $\sim 85 \%$ of the lipid species to become solid at $20^{\circ} \mathrm{C}$. Oguri et al. ${ }^{13}$ showed the formation of cholesterol-rich domains in CerNS24/FFA16/Chol and CerNS24/FFA24/ Chol mixtures after two heating-and-cooling cycles from 20 to $90{ }^{\circ} \mathrm{C}$, followed by a $10 \mathrm{~h}$ incubation at $37{ }^{\circ} \mathrm{C}$. In these samples, FFA24 mixed relatively well with ceramide and cholesterol, whereas the sample containing the shorter chain FFA16 showed phase separation after hydration. A spinodal decomposition has been proposed as a possible mechanism for the formation of lipid domains in SC model mixtures containing stearic acid (FFA18), a minor component of the SC lipid matrix. ${ }^{19}$ In this model, the formation of domains is associated with diffusion of lipid molecules, leading to a continuous change in composition of the phases during the phase separation. $^{20,21}$

The formation of lipid domains was reported primarily from X-ray diffraction, solid-state NMR, and vibrational spectroscopy studies. Phase separations were inferred by X-ray diffraction and solid-state NMR on the basis of the coexistence of the signatures of at least two different phases. For example, smalland wide-angle $\mathrm{X}$-ray diffraction patterns reported the presence of two crystalline structures in human SC, one with orthorhombic and the other one with a hexagonal lipid chain packing. ${ }^{4,22,23}$ Similarly, ${ }^{2} \mathrm{H}$ NMR allows describing the phase behavior of a deuterated species in a complex lipid mixture. Different mixtures of identical composition that include different deuterated components (generally referred to as mirror mixtures) can be used to assess the formation of domains. $^{14,18,24}$

Direct visualization of lipid domains in SC model membranes has been reported with the use of atomic force microscopy (AFM), fluorescence microscopy, and Raman microspectroscopy. ${ }^{25-31}$ For example, Norlén et al. ${ }^{25}$ showed, using AFM, that a monolayer formed from extracted SC total ceramides, cholesterol, and a mixture of FFA (length varying between $\mathrm{C} 20$ and $\mathrm{C} 30$ ) resulted in the formation of domains with a height difference of $\sim 10 \AA$. Similarly, ten Grotenhuis et al. ${ }^{29}$ observed, using AFM, that monolayers formed with BBCer mixed with cholesterol and FFA16 or FFA24 displayed the presence of domains with a thickness difference between the two phases of $6 \AA$, in the case of FFA16, and of $13 \AA$, in the case of FFA24. Using fluorescence microscopy, Plasencia et $a l^{26}$ studied the formation of lipid domains on a mixture composed of a Cer/Chol fraction extracted from human SC, and FFAs with lengths varying between $\mathrm{C} 20$ to $\mathrm{C} 30$. The different partitioning coefficient of two fluorescent probes $\left(1,1^{\prime}\right.$-dioctadecyl-3,3,3', $3^{\prime}$-tetramethylindocarbocyanine perchlorate $\left(\mathrm{DiI}_{\mathrm{C} 18}\right)$ and 6-lauroyl-2-(N,N-dimethylamino) naphthalene (Laurdan)) allowed to identify the coexistence of two solid/gel phases at the micrometer scale. Raman microspectroscopy has been also used for the visualization of lipid domains in SC model membranes. Raman chemical imaging can describe the local lipid composition of voxels and therefore the heterogeneity of SC lipid model mixtures. Generally, the introduction of a deuterated species facilitates this analysis because of the isotopic effect; the most common approach is the introduction of a lipid with deuterated chains as the $\mathrm{CH}$ elongation $(\nu \mathrm{CH})$ (between 2780 and $3100 \mathrm{~cm}^{-1}$ ) and the $\mathrm{CD}$ elongation $(\nu \mathrm{CD})$ (between 2000 and $2250 \mathrm{~cm}^{-1}$ ) are easily resolved in the Raman spectra. ${ }^{32}$ Percot and Lafleur ${ }^{27}$ showed the formation of domains enriched in the individual components in an equimolar mixture of BBCer, FFA16, and cholesterol using Raman microspectroscopy. The size of these domains exceeded $10 \mu \mathrm{m}$. Subsequent studies of a mixture of CerNS16, FFA16, and cholesterol showed that the lipids were mainly phase-separated despite the hydrophobic matching, with CerNS16- and FFA16-enriched crystalline-phase domains being detected. ${ }^{14}$

The methylene deformation region of infrared spectra is also used as a tool to determine phase separations. This region is sensitive to the symmetry of lipid chain packing in the solid phase. $^{20,33}$ A splitting of this band is observed for chains arranged in an orthorhombic geometry because of interchain vibrational coupling. ${ }^{2}$ This coupling occurs only if the methylene deformation vibrations of adjacent chains have similar frequencies. As the $\mathrm{CH}_{2}$ deformation $\left(\delta \mathrm{CH}_{2}\right)(\sim 1450$ $\left.\mathrm{cm}^{-1}\right)$ and the $\mathrm{CD}_{2}$ deformation $\left(\delta \mathrm{CD}_{2}\right)\left(\sim 1090 \mathrm{~cm}^{-1}\right)$ do not have the same frequency because of the isotopic effect, well-mixed hydrogenated and deuterated species in a structure with orthorhombic chain packing show a reduced splitting of the methylene deformation bands (both $\delta \mathrm{CH}_{2}$ and $\delta \mathrm{CD}_{2}$ ). Therefore, the magnitude of the splitting is a parameter describing the size and purity of the lipid domains, with the maximum splitting obtained for domains that include 100 molecules or more. ${ }^{20}$ This approach has been often used to characterize domains formation in SC lipid model mixtures because of their orthorhombic chain packing, similar to that of native SC lipid phase. ${ }^{28,33-35}$

In this paper, we analyze the influence of the chain length in Cer and FFA, two major components of SC lipid matrix, on the mixing properties of SC model membranes. Raman microspectroscopy and infrared spectroscopy were used to study the lipid spatial organization. By using these techniques, we found that the combination of very long chain FFA and Cer resulted in the formation of a highly homogeneous lipid structure, a feature that is believed to be essential for skin impermeability.

\section{MATERIALS AND METHODS}

2.1. Materials. N-lignoceroyl-D-erythro-sphingosine (CerNS24, $>99 \%$ ), $N$-palmitoyl-D-erythro-sphingosine (CerNS16, >99\%), and cholesterol (99\%) were purchase from Avanti Polar Lipids (Alabaster, AL, USA). Palmitic acid (FFA16, >99\%), stearic acid (FFA18, >99\%), arachidic acid (FFA20), lignoceric acid (FFA24, >99\%), ethylenediaminetetraacetic acid (EDTA, 99\%), sodium acetate (>99\%), and deuterium-depleted water $(>99 \%)$ were obtained from Sigma Chemical Co. (St. Louis, MO, USA). Perdeuterated FFA16- $d_{31}$ $(>99 \%)$, FFA20- $d_{39}(>98 \%)$, and FFA24- $d_{47}(>98 \%)$ were obtained 
from CDN Isotopes (Pointe-Claire, QC, Canada). Sodium chloride (>99\%) was purchased from Amresco (Solon, OH, USA). Methanol (spectrograde) and benzene (high purity) were obtained from American Chemicals Ltd. (Montréal, QC, Canada).

2.2. Mixtures Preparation. In this study, ternary lipid mixtures with CerNS and FFA with different length were prepared: CerNS16/ FFA16- $d$ /Chol, CerNS16/FFA24- $d$ /Chol, CerNS24/FFA16- $d /$ Chol, CerNS24/FFA20-d/Chol, and CerNS24/FFA24-d/Chol. The three lipid species (Cer, FFA, and cholesterol) were in an equimolar ratio. In addition, two samples containing an FFA mixture were prepared: in these, the FFA fraction was composed of FFA24- $d_{47}$, FFA20, FFA18, and FFA16, in a molar ratio of 84.6:8.75:4.57:2.05, a distribution representative of the SC FFA composition. ${ }^{36,37}$ The FFA mixture is labeled using FFAs, and therefore, the mixtures are identified by CerNS16/FFAs/Chol and CerNS24/FFAs/Chol.

The lipids were individually dissolved in a benzene/methanol (7:3 $\mathrm{v} / \mathrm{v}$ ) solution and aliquots with appropriate volume were mixed to obtain the desired proportions. The samples were freeze-dried, and the resulting lipid powders were hydrated with acetate buffer $(50 \mathrm{mM}$, $\mathrm{pH} 5$ ) following the procedure proposed by Oguri et al. ${ }^{13}$ Briefly, the samples underwent a thermal treatment consisting of two heating/ cooling cycles from 20 to $90^{\circ} \mathrm{C}$, with an equilibration time of $10 \mathrm{~min}$ at each temperature. The buffer was added after the equilibration time following the second cycle. Finally, the samples were incubated at 37 ${ }^{\circ} \mathrm{C}$ for 7 days to favor a thermodynamically stable state. The data were then acquired at $33{ }^{\circ} \mathrm{C}$, representative of the epidermis temperature.

For the kinetics studies, the mixtures were subjected to the same two heating/cooling cycles and the Raman and IR measurements were launched immediately after the buffer addition. The data were recorded at $37{ }^{\circ} \mathrm{C}$, the temperature used during the equilibration process.

2.3. Raman Microspectroscopy Analysis. The samples (waxy solids) were placed on a $\mathrm{CaF}_{2}$ window, gently squeezed to obtain a relatively flat surface, and immersed in the acetate buffer to maintain complete hydration during the measurements. The temperature was regulated by a heating and cooling stage. The Raman spectra were obtained with a WITec Alpha 300 confocal Raman microscope (Ulm, Germany), equipped with a $63 \mathrm{X}$ immersion objective (NA 1.0), and a diffraction grating of 600 lines/mm. A $532 \mathrm{~nm}$ laser was used as excitation source with a $10 \mathrm{~mW}$ power at the sample surface. The focus was made at the lipid/buffer interface. With this setup, the depth of focus was estimated to $2 \mu \mathrm{m}$. All the images were obtained over a $30 \times 30 \mu \mathrm{m}^{2}$ area, with a lateral resolution of $0.67 \mu \mathrm{m} /$ pixel and an integration time of $5 \mathrm{~s}$ per spectrum, leading to a total acquisition time of $\sim 3 \mathrm{~h}$ per map. The spectra were treated to obtain the FFA molar proportion following a previously reported procedure. $^{38}$ In short, the areas of the $\nu \mathrm{CD}\left(2000-2250 \mathrm{~cm}^{-1}\right)$ and $\nu \mathrm{CH}\left(2780-3100 \mathrm{~cm}^{-1}\right)$ regions were considered proportional to, respectively, the $\mathrm{CD}$ and $\mathrm{CH}$ local concentration. The deuterated FFA molar proportion of each pixel, $X_{\mathrm{CD}}^{\mathrm{pix}}$, was estimated using:

$$
X_{\mathrm{CD}}^{\mathrm{pix}}=\left(\frac{1}{1+\frac{A_{\mathrm{CH}}^{\mathrm{pix}}}{A_{\mathrm{CD}}^{\mathrm{pix}}} \frac{[\mathrm{CH}]_{\text {mix }} A_{\mathrm{CD}}^{\mathrm{av}}}{[\mathrm{Cu}} A_{\mathrm{CH}}^{\mathrm{av}}}\right) \times 100
$$

where $A_{\mathrm{CD}}^{\mathrm{pix}}$ and $A_{\mathrm{CH}}^{\mathrm{pix}}$ are the areas of the $\nu \mathrm{CD}$ region and the $\nu \mathrm{CH}$ region of the spectrum of a given pixel, $A_{\mathrm{CD}}^{\mathrm{av}}$ and $A_{\mathrm{CH}}^{\mathrm{av}}$ are the areas of the $\nu \mathrm{CD}$ region and the $\nu \mathrm{CH}$ region of the spectrum averaged from the whole region, and $[\mathrm{CD}]_{\text {mix }}$ and $[\mathrm{CH}]_{\text {mix }}$ are the proportions of the deuterated and hydrogenated species in the mixture, respectively. The false-color distribution maps and histograms were generated using OriginPro software.

In the kinetics studies, Raman spectra were collected from the samples right after the buffer addition (time $=0$ ). For a given kinetics experiment, all the Raman measurements were acquired from the same $30 \times 30 \mu \mathrm{m}^{2}$ spot, except those after 7 days, which were recorded in the same vicinity of the same sample. The integration time was set to $3 \mathrm{~s}$ per spectrum, leading to a total acquisition time of $\sim 2 \mathrm{~h}$. The temperature was adjusted to $37{ }^{\circ} \mathrm{C}$ to reproduce the conditions of incubation.

2.4. IR Analysis. The IR spectra were acquired using a Thermo Nicolet 4700 spectrometer. The lipid mixtures were analyzed in transmission mode, putting the samples (after the two heating/ cooling cycles from 20 to $90{ }^{\circ} \mathrm{C}$ ) and $\sim 25 \mu \mathrm{L}$ of acetate buffer between two $\mathrm{CaF}_{2}$ windows separated by a $5 \mu \mathrm{m}$-thick Teflon spacer. The windows were sealed with silicone grease to maintain complete hydration during the measurements. The temperature was adjusted to $37{ }^{\circ} \mathrm{C}$, using a temperature-controlled brass sample holder. The spectra were recorded every $20 \mathrm{~min}$ during $24 \mathrm{~h}$, co-adding 64 scans with a nominal spectral resolution of $0.1 \mathrm{~cm}^{-1}$.

\section{RESULTS AND DISCUSSION}

Figure 1 shows the Raman spectra of two different lipid mixtures. The spectra were obtained from averaging those

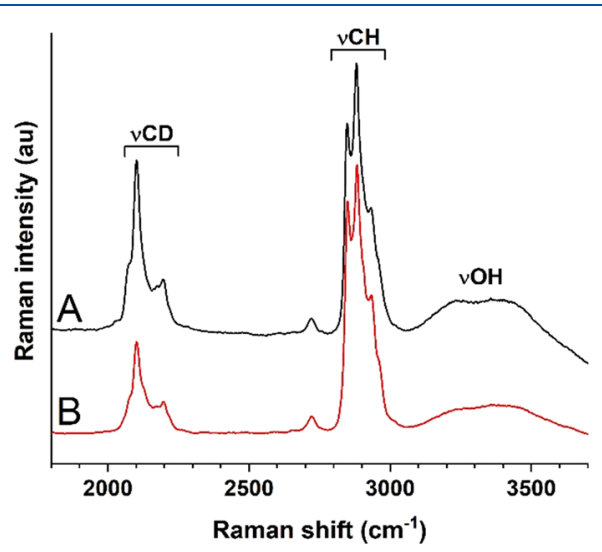

Figure 1. Raman spectra of two investigated mixtures: (A) CerNS24/ FFA24- $d /$ Chol and (B) CerNS24/FFA16- $d /$ Chol.

recorded for the Raman imaging, and therefore, they are representative of the entire surface.

The $\nu \mathrm{CD}$ region is assigned to the fully deuterated FFA chain. This signal was mainly composed of the antisymmetric methylene $\left(\mathrm{CD}_{2}\right)$ and the methyl $\left(\mathrm{CD}_{3}\right)$ stretching modes, at 2102 and $2196 \mathrm{~cm}^{-1}$, respectively. The most intense signal of the spectra corresponds to the $(\nu \mathrm{CH}$ region. The symmetric and antisymmetric $\mathrm{CH}_{2}$ stretching modes were located at 2848 and $2881 \mathrm{~cm}^{-1}$, respectively, while the symmetric $\mathrm{CH}_{3}$ stretching mode appeared at $2933 \mathrm{~cm}^{-1}$. The shoulder located at around $2960 \mathrm{~cm}^{-1}$ corresponded to the antisymmetric $\mathrm{CH}_{3}$ stretching. This region included contributions from CerNS and cholesterol. The broad band located between 3080 and 3650 $\mathrm{cm}^{-1}$ mainly corresponded to the $\mathrm{O}-\mathrm{H}$ stretching from water.

In order to investigate the FFA spatial distribution in the mixtures, we recorded 2025 Raman spectra over a $30 \times 30 \mu \mathrm{m}^{2}$ surface of the samples, incubated at $37{ }^{\circ} \mathrm{C}$ for 7 days. The spectra were treated to obtain false-color distribution maps of the FFA molar proportion (see section 2.3). The resulting chemical images and their corresponding histograms are presented in Figure 2.

The FFA distribution over the investigated areas was very similar for the CerNS16/FFA16- $d$ /Chol, CerNS16/FFAs/ Chol (Figure S1, Supporting Information), CerNS16/FFA24$d / \mathrm{Chol}$, and CerNS24/FFA16- $d /$ Chol samples. In these mixtures, FFA was unevenly distributed, forming after one week of incubation enriched domains on the $\sim 10 \mu \mathrm{m}$ scale, if the criterion for defining a lipid domain was a local molar proportion equal or greater than $42 \%$, which is $25 \%$ higher than the mixture molar proportion of $33 \%$. Some small regions 

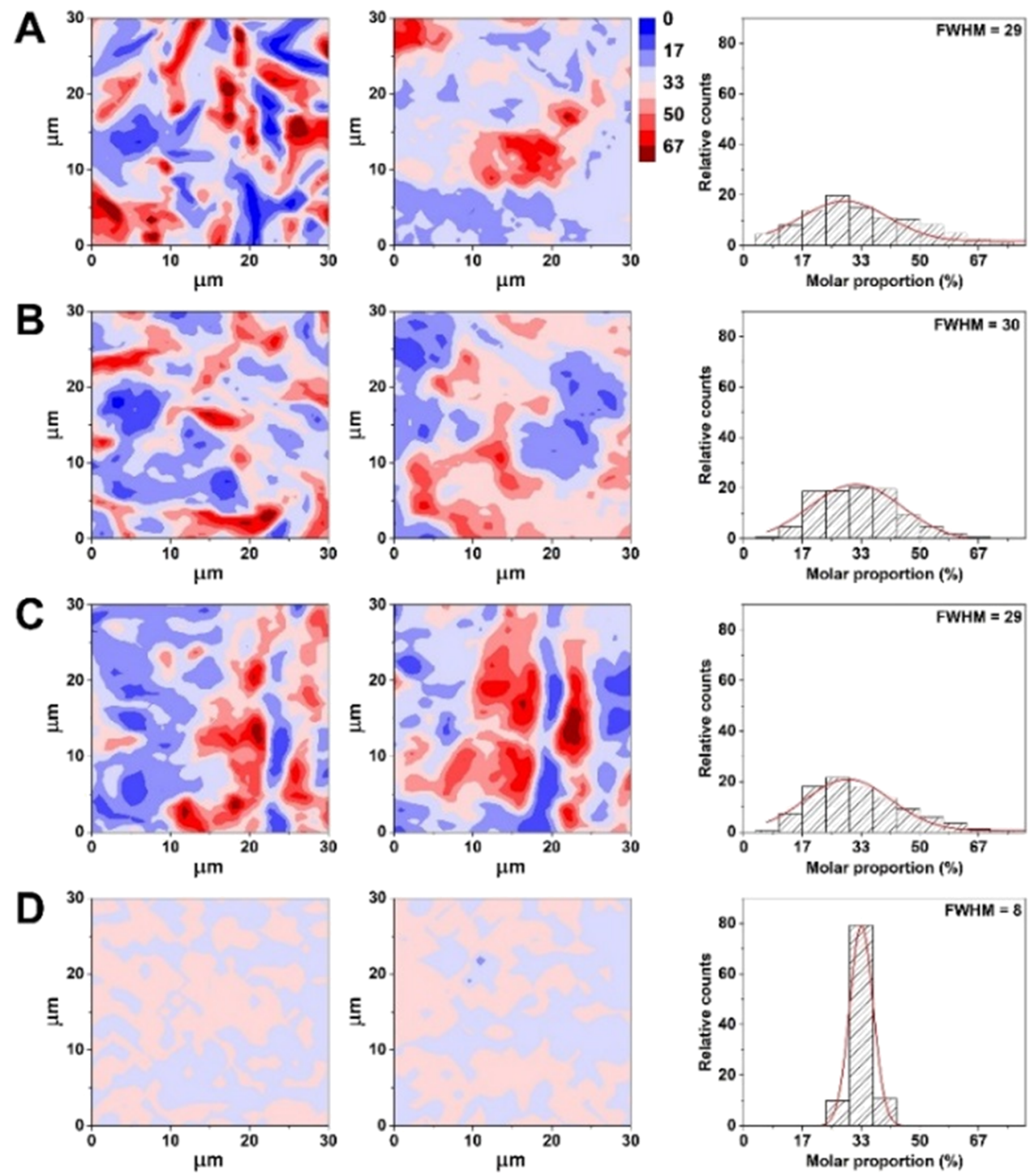

Figure 2. The first two columns present two typical Raman images whereas the third column is the corresponding histograms of (A) CerNS16/ FFA16- $d$ /Chol, (B) CerNS16/FFA24- $d /$ Chol, (C) CerNS24/FFA16- $d / C h o l$, and (D) CerNS24/FFA24- $d /$ Chol mixtures. The chemical maps represent the spatial distribution of the deuterated free fatty acid; dark blue represents depleted regions $(\sim 0 \%)$, while dark red represents enriched regions $(\geq 67 \%)$. The data were acquired at $33{ }^{\circ} \mathrm{C}$, after the equilibration process described in the Materials and Methods section. The histograms combined the data of the two Raman images from each mixture and therefore represent 4050 pixels each. The red curves in the histograms represent the best-fit normal distributions.

reached a high FFA content, exceeding a local molar proportion of $67 \%$. Besides, there were some FFA-depleted regions where the molar proportion was under $25 \%$, reaching at some places values as low as $17 \%$. The full width at halfmaximum (FWHM) of the normal distribution of the local FFA content was $\sim 30 \%$, indicating a considerable variation of local lipid composition or, in other words, a significant heterogeneous lipid spatial distribution.

In contrast, the CerNS24/FFA24- $d$ /Chol (Figure 2D) and CerNS24/FFAs/Chol (Figure S1, Supporting Information) mixtures showed a considerably more homogeneous FFA24-d distribution. In these samples, the molar proportion only varied between 25 and $42 \%$ over the entire surface. The distributions were centered at $33 \%$ and could be nicely reproduced by a normal distribution. The FWHM was $8 \%$, indicating that FFA24 was homogeneously distributed over the surface. These results indicate that the presence of very long FFA chains, as existing in native SC, favors the mixing of the lipid components when the main ceramide is CerNS24. This conclusion is in agreement with a previous study that indicated that the very long FFA24 promotes lipid mixing in matrices formed by cholesterol and CerNS24. ${ }^{13}$

We studied the kinetics of FFA domain formation in three samples: CerNS24/FFA16- $d /$ Chol, CerNS24/FFA20- $d /$ Chol, and CerNS24/FFA24- $d$ /Chol. These mixtures included, in an equimolar ratio, the same type of Cer (CerNS24), cholesterol, but three different FFAs: the short-chain FFA16, the long chain FFA20, and the very long chain FFA24. The evolution of the FFA distribution was followed by Raman microspectroscopy, as shown in Figure 3.

As described above, the samples were submitted to two heating/cooling cycles from 20 to $90{ }^{\circ} \mathrm{C}$, quenched after the last high-temperature incubation (they were cooled rapidly by placing the samples at $90{ }^{\circ} \mathrm{C}$ on a metal support that was at $10-15{ }^{\circ} \mathrm{C}$; the temperature decrease took place in less than 1 min) and then immersed in acetate buffer at $37{ }^{\circ} \mathrm{C}$. The 

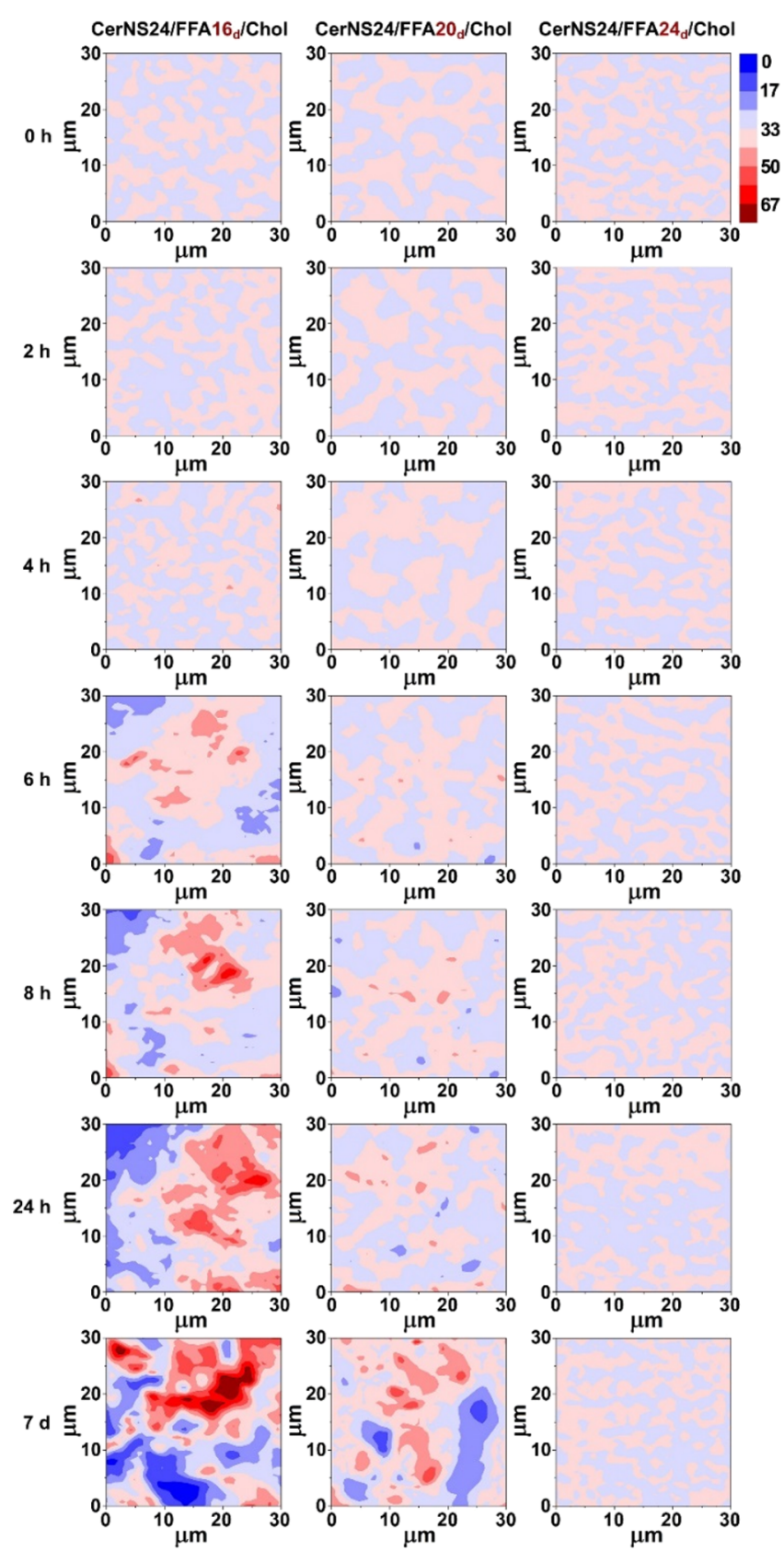

Figure 3. Raman images describing the time evolution of the lipid mixing properties during hydration; the images were collected over an area $30 \times 30 \mu \mathrm{m}^{2}$. The 7-day images were not collected from the very same region as the previous images. The chemical maps represent the spatial distribution of the deuterated free fatty acid; dark blue represents depleted regions $(\sim 0 \%)$, while dark red represents enriched regions $(\geq 67 \%)$. The data were acquired at $37{ }^{\circ} \mathrm{C}$, the temperature used for the equilibration process as described in the Materials and Methods section.

measurements were launched immediately after, and the time was set to 0 . Initially, the mixtures showed a highly homogeneous surface, with the molar proportion varying from 25 to $42 \%(+/-25 \%$ of the theoretical $33 \%)$. In the first 2 $\mathrm{h}$ of incubation, no significant changes were observed in the lipid spatial distribution. The CerNS24/FFA16- $d /$ Chol mixture showed the formation of small FFA16- $d$ domains after $4 \mathrm{~h}$ of incubation; the local molar proportion could be above $42 \%$. The lipid segregation became more and more pronounced with time. After 7 days, the Raman images showed a significant heterogeneity, with large regions enriched or depleted in FFA. The molar proportion of the most FFA16enriched domains increased to values over $67 \%$, while the depleted zones showed values under $13 \%$.

The CerNS24/FFA20- $d /$ Chol mixture remained homogeneous for $\sim 6 \mathrm{~h}$ of incubation. Subsequently, small heterogeneities were observed, and their size and the extent of FFAenrichment grew progressively as a function of time. After a 7 day incubation, FFA20-enriched domains displayed a local FFA molar proportion over $50 \%$, depleted regions had a local molar proportion of $17 \%$, and the dimensions of these domains were up to $10 \mu \mathrm{m}$. However, the heterogeneity of this mixture was generally more limited than that observed for the CerNS24/FFA16- $d$ /Chol mixture. In contrast, the CerNS24/ FFA24- $d$ /Chol mixture remained homogeneous, as characterized by Raman microimaging, even after 7 days of incubation.

A quantitative approach to probe domain formation kinetics is measuring the mean deviation of the local molar proportion as a function of time (Figure 4). The mean of the absolute

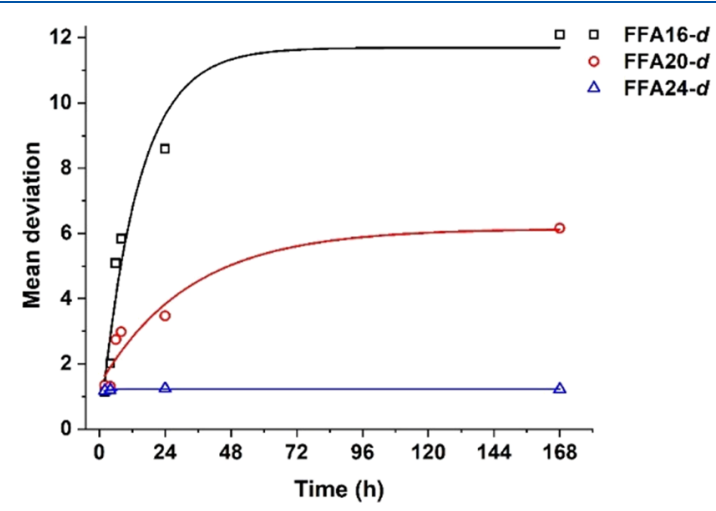

Figure 4. Kinetics of the evolution of the mean deviation in CerNS24/FFA- $d$ /Chol mixtures for different FFA chain length.

deviations of the local molar proportions from the average value describes how far, on average, the values are from the overall molar proportion $(33 \%)$ or, in other words, how (hetero)homogeneous the mixture is.

Two observations are derived from these results. First, after a week of incubation ( $168 \mathrm{~h})$, the mixture remained relatively homogeneous when the present fatty acid was FFA24, while there were significant local composition variations with FFA20, and these were even more pronounced with FFA16. Second, the kinetics of the phase separation of the mixture with FFA16 was faster than that of the mixture with FFA20; the mean deviation was practically constant for the mixture with FFA24, and therefore, no kinetics parameter could be inferred. The characteristic time obtained from fitting a negative exponential function was $33 \pm 17 \mathrm{~h}$ for the mixture with FFA16 whereas it was $14 \pm 5 \mathrm{~h}$ for the mixture with FFA20.

It should be pointed out that the initial homogeneous mixtures were obtained only when the samples were quenched from $90^{\circ} \mathrm{C}$. When the ternary mixtures were cooled at a slower rate, the samples containing FFA20 (the melting point, $T_{\mathrm{m}}$, of pure FFA20 is $\left.75{ }^{\circ} \mathrm{C}\right)^{39}$ or FFA16 $\left(T_{\mathrm{m}}=62^{\circ} \mathrm{C}\right),{ }^{39}$ displayed FFA-depleted and FFA-enriched domains (for reference, the $T_{\mathrm{m}}$ of CerNS24 is $\left.82^{\circ} \mathrm{C}\right) .{ }^{40}$ In some extreme cases, almost pure FFA microscopic domains were observed. The quenching 


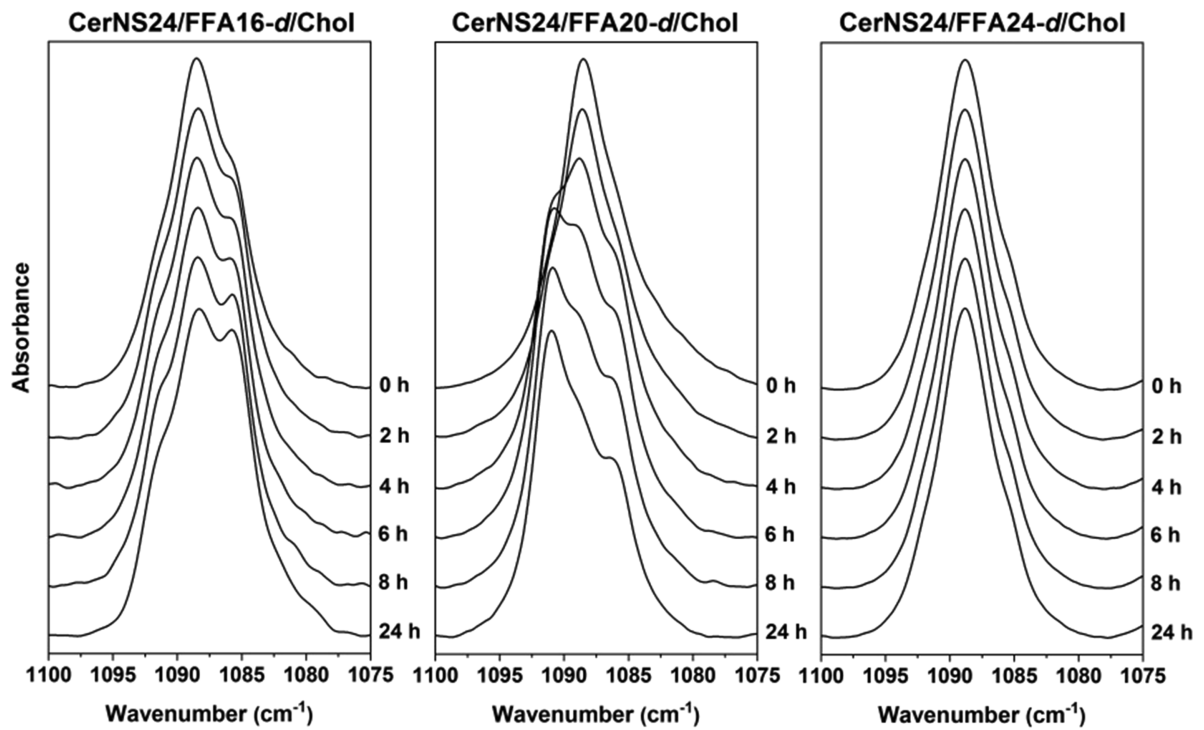

Figure 5. Evolution of the $\mathrm{CD}_{2}$ deformation band during the first $24 \mathrm{~h}$ of incubation at $37^{\circ} \mathrm{C}$.

\section{$\mathrm{CH}_{2}$ deformation region}

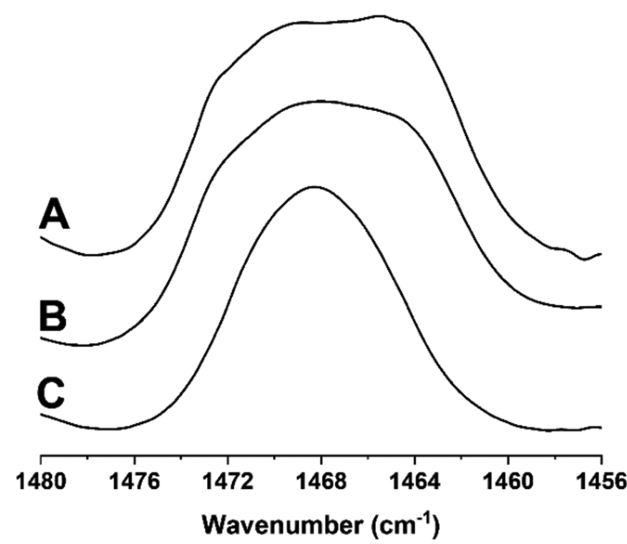

$C D_{2}$ deformation region

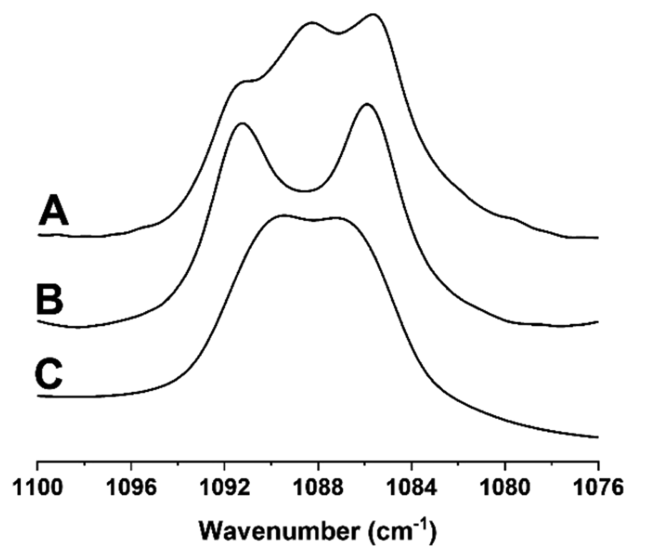

Figure 6. $\delta \mathrm{CH}_{2}$ and $\delta \mathrm{CD}_{2}$ regions recorded at $33{ }^{\circ} \mathrm{C}$ after a 7 day incubation at $37{ }^{\circ} \mathrm{C}$. (A) CerNS24/FFA16- $d / \mathrm{Chol}$, (B) CerNS24/FFA20-d/ Chol, and (C) CerNS24/FFA24- $d /$ Chol.

was the selected approach to obtain a reproducible behavior. The mixture containing CerNS24 and FFA2 $4\left(T_{\mathrm{m}}=84{ }^{\circ} \mathrm{C}\right)^{39}$ appeared to remain homogeneous even at a slower cooling rate.

In order to substantiate the ability of chain length to modulate lipid mixing and domain formation, we analyzed the methylene deformation $\left(\delta \mathrm{CD}_{2}\right)$ region of the infrared spectra of the mixtures (Figure 5). As described above, this region is often used to assess the extent of lipid mixing. ${ }^{33,41}$

The width of the $\delta \mathrm{CD}_{2}$ mode of the CerNS24/FFA16- $d /$ Chol mixture, measured at the $50 \%$ of the band maximum, increased from $6.5 \mathrm{~cm}^{-1}$ at $t=0$ to $8.1 \mathrm{~cm}^{-1}$ after $24 \mathrm{~h}$ incubation. This evolution suggests the progressive formation of FFA16- $d$-enriched orthorhombic lateral structures, in agreement with FFA16- $d$-enriched domains that were observed by Raman imaging. The CerNS24/FFA20- $d /$ Chol mixture showed a similar behavior: the width of its $\delta \mathrm{CD}_{2}$ mode varied from $5.5 \mathrm{~cm}^{-1}$ at $t=0$ to $7.2 \mathrm{~cm}^{-1}$ after a $24 \mathrm{~h}$ incubation. In contrast, the $\delta \mathrm{CD}_{2}$ region of the CerNS24/FFA24- $d /$ Chol displayed a maximum located at $1088.8 \mathrm{~cm}^{-1}$ and a $\delta \mathrm{CD}_{2}$ band width of $5.0 \mathrm{~cm}^{-1}$. This profile remained practically unaltered during the first $24 \mathrm{~h}$ of incubation. This result is in agreement with a previous analysis reporting that a mixture with the same composition displayed a $\delta \mathrm{CD}_{2}$ band width of $3.8 \mathrm{~cm}^{-1}$ at 36 ${ }^{\circ} \mathrm{C}$, after a $10 \mathrm{~h}$ incubation. ${ }^{42}$ It should be pointed out that the kinetics of the domain formation is reminiscent of that observed by Raman imaging, even though the techniques are not sensitive to the same length scale.

After a 7 day incubation at $37^{\circ} \mathrm{C}$, the infrared spectra were recorded at $33{ }^{\circ} \mathrm{C}$ (Figure 6). The $\delta \mathrm{CD}_{2}$ region of the mixtures showed a splitting for every mixture, indicative of the formation of FFA-enriched domains with an orthorhombic chain packing. ${ }^{33}$ In addition to a central component at 1088.4 $\mathrm{cm}^{-1}$, the CerNS24/FFA16- $d /$ Chol mixture showed split components at 1091.4 and $1085.5 \mathrm{~cm}^{-1}$, with the positions being determined on the Fourier self-deconvoluted spectra. The CerNS24/FFA20- $d$ /Chol mixture also displayed the two components, located at 1091.3 and $1085.9 \mathrm{~cm}^{-1}$. The CerNS24/FFA24- $d$ /Chol samples also showed a splitting, but it was considerably smaller, with the components being at 1089.8 and $1086.7 \mathrm{~cm}^{-1}$. The $\delta \mathrm{CH}_{2}$ mode can also display a band splitting associated with interchain coupling. ${ }^{20,43}$ In this case, the splitting was not as well defined as in the $\delta \mathrm{CD}_{2}$ region because of the overlap with the spectral contributions from 
cholesterol and the amide II band of ceramide. However, the width of the $\delta \mathrm{CH}_{2}$ mode, measured at the $50 \%$ of the band maximum was $7.8 \mathrm{~cm}^{-1}$ for the mixture containing FFA24, whereas it was $11.7 \mathrm{~cm}^{-1}$ for the samples containing FFA16 and FFA20, suggesting a more pronounced splitting for FFA with a shorter acyl chain. The presence of the $\delta \mathrm{CH}_{2}$ and the $\delta \mathrm{CD}_{2}$ band splittings in the three mixtures indicates the existence of FFA- $d$-enriched domains with orthorhombic chain packing. However, the size and/or the enrichment of these domains was significantly more limited in the case of the mixture containing the very-long chain FFA24.

The present results demonstrate that CerNS and FFA bearing very long saturated acyl chains (C24) lead to mixtures considerably more homogeneous than those that include CerNS or FFA with a C16 or C20 chain. The homogeneity of the mixtures was assessed on the micrometer scale by Raman chemical imaging and at the molecular level from the IR spectroscopic analysis. The results clearly establish the key role of lipid chain length for the formation of a homogeneous lipid matrix. Intermolecular interactions between the components of SC lipid matrix include $\mathrm{H}$-bonding at the head group level as well as van der Waals interactions at the chain level. ${ }^{44-46}$ Experimental and theoretical studies have shown the importance of $\mathrm{H}$ bonds in the structure of SC lipids. ${ }^{4-50}$ Extensive phase separations were observed in the CerNS16/ FFA16/Chol mixture, ${ }^{14}$ a mixture for which the hydrophobic matching between the components is optimal. This finding suggests that interfacial intermolecular interactions are a driving factor of this phase separation. The strength of CerCer H-bonds has been estimated, from $a b$ initio and density functional theory (DFT) methods, to vary between 20 and 62 $\mathrm{kJ} / \mathrm{mol}^{47}$ these should contribute to phase separations if they are more stable than those between ceramide and FFA and/or cholesterol. Actually, molecular dynamics simulations of CerNS24/FFA24/Chol mixtures showed that H-bonding between Cer-Cer pairs is dominating, if the $\mathrm{H}$-bond involving water are not considered. ${ }^{48-50}$ In these studies, the lipid distribution was found to be homogeneous, except that molecular clusters involving about five molecules with a slight enrichment in CerNS were reported in one study. ${ }^{50}$ Very long acyl chains should lead to a relative increase of the contribution of van der Waals interactions; the interaction energy between $2 \mathrm{CH}_{2}$ of closely packed chains is estimated to $-3.5 \mathrm{~kJ} / \mathrm{mol}^{51}$ it is therefore evaluated that attractive interchain van der Waals interactions would increase by about $28 \mathrm{~kJ} / \mathrm{mol}$ for an acyl chain going from C16 to C24. It is proposed that this contribution favors lipid mixing in the SC lipid phase.

The relatively slow evolution of the phase-separated morphology is a key point that should be taken into consideration when determining and comparing the properties of SC model mixtures. It is not established which equilibration protocol reproduces in vivo conditions best, but it is certain that different equilibration protocols can lead to considerable differences in mixing properties as well as in all the properties associated with domain formation, such as permeability.

The present results obtained on SC model mixtures indicate that chain elongation favors the formation of a homogeneous lipid phase, a feature that is required for a skin barrier that is functioning properly. The lipid chain elongation in skin involves a complex enzymatic machinery and a considerable expenditure of energy; chains are elongated, two carbon groups at a time, using a four-reaction cycle involving several enzymes including fatty acid elongases. ${ }^{52}$ Mice deficient in elongases died shortly after birth; their SC lipid lamellae were considerably thinned and their skin barrier was largely impaired, showing considerable increased trans-epidermal water loss. ${ }^{53}$ Analogous observations have been done on model systems. Previous studies ${ }^{16,42}$ showed that model membranes prepared from CerNS, FFA24, cholesterol, and cholesterol sulfate (CholS) displayed a limited permeability, measured by water loss as well as theophylline and indomethacin permeability when CerNS24 was used compared to a mixture including CerNS16 or CerNS with shorter acyl chains. ${ }^{16,54}$ Shorter-chain CerNS are less miscible with FFA24 than CerNS24, and these different mixing properties were proposed to be associated with the permeability variations. It should be noted that very long chain lipids also lead to thicker bilayers, and this could indeed contribute to limit the permeability.

The very long chains of SC lipids, which are resulting from the elaborate machinery for chain elongation, constitute a key structural element favoring the formation of a homogeneous phase and are therefore proposed to contribute to the formation of an efficient skin barrier.

\section{ASSOCIATED CONTENT}

\section{SI Supporting Information}

The Supporting Information is available free of charge at https://pubs.acs.org/doi/10.1021/acs.langmuir.0c02305.

Typical Raman chemical images and the corresponding histograms obtained from CerNS16/FFAs/Chol and CerNS24/FFAs/Chol mixtures (PDF)

\section{AUTHOR INFORMATION}

\section{Corresponding Author}

Michel Lafleur - Department of Chemistry, Université de Montréal, Montréal QC H3C 3J7, Canada; (1) orcid.org/ 0000-0003-3868-9803; Phone: (514) 343-5936;

Email: michel.lafleur@umontreal.ca; Fax: (514) 343-7586

\section{Authors}

Adrian Paz Ramos - Department of Chemistry, Université de Montréal, Montréal QC H3C 3J7, Canada

Joke A. Bouwstra - Drug Delivery Technology, Leiden Academic Centre for Drug Research, Leiden 2333 CC, The Netherlands

Complete contact information is available at: https://pubs.acs.org/10.1021/acs.langmuir.0c02305

\section{Notes}

The authors declare no competing financial interest.

\section{ACKNOWLEDGMENTS}

The authors thank the Natural Sciences and Engineering Research Council (NSERC) of Canada for the financial support. A.P.R. thanks the Fonds Québécois de la Recherche sur la Nature et les Technologies (FQR-NT) for his Doctoral Research Scholarship for Foreign Students. This work was also funded by the FQRNT through its financial support to the Centre québécois sur les matériaux fonctionnels (CQMF).

\section{REFERENCES}

(1) Harding, C. R. The stratum corneum: structure and function in health and disease. Dermatol. Ther. 2004, 17, 6-15. 
(2) Johnson, M. E.; Blankschtein, D.; Langer, R. Evaluation of solute permeation through the stratum corneum: lateral bilayer diffusion as the primary transport mechanism. J. Pharm. Sci. 1997, 86, 11621172.

(3) White, S. H.; Mirejovsky, D.; King, G. I. Structure of lamellar lipid domains and corneocyte envelopes of murine stratum corneum An x-ray diffraction study. Biochemistry 1988, 27, 3725-3732.

(4) Bouwstra, J. A.; Gooris, G. S.; van der Spek, J. A.; Bras, W. Structural investigations of human stratum corneum by small-angle $\mathrm{x}$ ray scattering. J. Invest. Dermatol. 1991, 97, 1005-1012.

(5) Pilgram, G. S. K.; Marjolein Engelsma-van Pelt, A.; Koerten, H. K.; Bouwstra, J. A. Electron diffraction provides new information on human stratum corneum lipid organization studied in relation to depth and temperature. J. Invest. Dermatol. 1999, 113, 403-409.

(6) Choe, C.; Schleusener, J.; Lademann, J.; Darvin, M. E. Human skin in vivo has a higher skin barrier function than porcine skin ex vivo - comprehensive Raman microscopic study of the stratum corneum. J. Biophotonics. 2018, 11, e201700355.

(7) de Jager, M. W.; Gooris, G. S.; Dolbnya, I. P.; Bras, W.; Ponec, M.; Bouwstra, J. A. Novel lipid mixtures based on synthetic ceramides reproduce the unique stratum corneum lipid organization. J. Lipid Res. 2004, 45, 923-932.

(8) Bouwstra, J. A.; Gooris, G. S.; Dubbelaar, F. E. R.; Ponec, M. Phase behavior of stratum corneum lipid mMixtures based on human ceramides: the role of natural and synthetic ceramide 1 . J. Invest. Dermatol. 2002, 118, 606-617.

(9) Langner, M.; Hui, S. W. Dithionite penetration through phospholipid bilayers as a measure of defects in lipid molecular packing. Chem. Phys. Lipids 1993, 65, 23-30.

(10) Clerc, S. G.; Thompson, T. E. Permeability of dimyristoyl phosphatidylcholine/dipalmitoyl phosphatidylcholine bilayer membranes with coexisting gel and liquid-crystalline phases. Biophys. J. 1995, 68, 2333-2341.

(11) Swartzendruber, D. C.; Manganaro, A.; Madison, K. C.; Kremer, M.; Wertz, P. W.; Squier, C. A. Organization of the intercellular spaces of porcine epidermal and palatal stratum corneum: a quantitative study employing ruthenium tetroxide. Cell Tissue Res. 1995, 279, 271-276.

(12) Chen, X.; Kwak, S.; Lafleur, M.; Bloom, M.; Kitson, N.; Thewalt, J. Fatty acids influence "solid" phase formation in models of stratum corneum intercellular membranes. Langmuir 2007, 23, 55485556.

(13) Oguri, M.; Gooris, G. S.; Bito, K.; Bouwstra, J. A. The effect of the chain length distribution of free fatty acids on the mixing properties of stratum corneum model membranes. Biochim. Biophys. Acta 2014, 1838, 1851-1861.

(14) Brief, E.; Kwak, S.; Cheng, J. T. J.; Kitson, N.; Thewalt, J.; Lafleur, M. Phase behavior of an equimolar mixture of $\mathrm{n}$-palmitoyl-derythro-sphingosine, cholesterol, and palmitic acid, a mixture with optimized hydrophobic matching. Langmuir 2009, 25, 7523-7532.

(15) Školová, B.; Hudská, K.; Pullmannová, P.; Kováčik, A.; Palát, K.; Roh, J.; Fleddermann, J.; Estrela-Lopis, I.; Vávrová, K. Different phase behavior and packing of ceramides with long (C16) and very long (C24) acyls in model membranes: infrared spectroscopy using deuterated lipids. J. Phys. Chem. B 2014, 118, 10460-10470.

(16) Pullmannová, P.; Pavlíková, L.; Kováčik, A.; Sochorová, M.; Školová, B.; Slepička, P.; Maixner, J.; Zbytovská, J.; Vávrová, K. Permeability and microstructure of model stratum corneum lipid membranes containing ceramides with long $(\mathrm{C} 16)$ and very long (C24) acyl chains. Biophys. Chem. 2017, 224, 20-31.

(17) Lafleur, M. Phase behaviour of model stratum corneum lipid mixtures: an infrared spectroscopy investigation. Can. J. Chem. 1998, 76, 1501-1511.

(18) Fenske, D. B.; Thewalt, J. L.; Bloom, M.; Kitson, N. Models of stratum corneum intercellular membranes: $2 \mathrm{H}$ NMR of macroscopically oriented multilayers. Biophys. J. 1994, 67, 1562-1573.

(19) Mendelsohn, R.; Selevany, I.; Moore, D. J.; Mack Correa, M. C.; Mao, G.; Walters, R. M.; Flach, C. R. Kinetic evidence suggests spinodal phase separation in stratum corneum models by IR spectroscopy. J. Phys. Chem. B 2014, 118, 4378-4387.

(20) Snyder, R. G.; Goh, M. C.; Srivatsavoy, V. J. P.; Strauss, H. L.; Dorset, D. L. Measurement of the growth kinetics of microdomains in binary n-alkane solid solutions by infrared spectroscopy. J. Phys. Chem. 1992, 96, 10008-10019.

(21) Gilbert, E. P.; Reynolds, P. A.; Thiyagarajan, P.; Wozniak, D. G.; White, J. W. Microphase separation kinetics in n-alkane mixtures. Phys. Chem. Chem. Phys. 1999, 1, 2715-2724.

(22) Bouwstra, J. A.; Gooris, G. S.; Cheng, K.; Weerheim, A.; Bras, W.; Ponec, M. Phase behavior of isolated skin lipids. J. Lipid Res. 1996, 37, 999-1011.

(23) Bouwstra, J. A.; Gooris, G. S.; Salomons-de Vries, M. A.; Van der Spek, J. A.; Bras, W. Structure of human stratum corneum as a function of temperature and hydration: a wide-angle X-ray diffraction study. Int. J. Pharm. 1992, 84, 205-216.

(24) Paz Ramos, A.; Lafleur, M. Chain length of free fatty acids influences the phase behavior of stratum corneum model membranes. Langmuir 2015, 31, 11621-11629.

(25) Norlén, L.; Gil, I. P.; Simonsen, A.; Descouts, P. Human stratum corneum lipid organization as observed by atomic force microscopy on Langmuir-Blodgett films. J. Struct. Biol. 2007, 158, $386-400$.

(26) Plasencia, I.; Norlén, L.; Bagatolli, L. A. Direct visualization of lipid domains in human skin stratum corneum's lipid membranes: effect of $\mathrm{pH}$ and temperature. Biophys. J. 2007, 93, 3142-3155.

(27) Percot, A.; Lafleur, M. Direct observation of domains in model stratum corneum lipid mixtures by Raman microspectroscopy. Biophys. J. 2001, 81, 2144-2153.

(28) Chen, H.-C.; Mendelsohn, R.; Rerek, M. E.; Moore, D. J. Effect of cholesterol on miscibility and phase behavior in binary mixtures with synthetic ceramide 2 and octadecanoic acid. Infrared studies. Biochim. Biophys. Acta 2001, 1512, 345-356.

(29) ten Grotenhuis, E.; Demel, R. A.; Ponec, M.; Boer, D. R.; van Miltenburg, J. C.; Bouwstra, J. A. Phase behavior of stratum corneum lipids in mixed Langmuir-Blodgett monolayers. Biophys. J. 1996, 71, $1389-1399$

(30) Mojumdar, E. H.; Gooris, G. S.; Groen, D.; Barlow, D. J.; Lawrence, M. J.; Demé, B.; Bouwstra, J. A. Stratum corneum lipid matrix: location of acyl ceramide and cholesterol in the unit cell of the long periodicity phase. Biochim. Biophys. Acta 2016, 1858, 19261934.

(31) Mojumdar, E. H.; Groen, D.; Gooris, G. S.; Barlow, D. J.; Lawrence, M. J.; Deme, B.; Bouwstra, J. A. Localization of cholesterol and fatty acid in a model lipid membrane: a neutron diffraction approach. Biophys. J. 2013, 105, 911-918.

(32) Mendelsohn, R.; Sunder, S.; Bernstein, H. J. Deuterated fatty acids as Raman spectroscopic probes of membrane structure. Biochim. Biophys. Acta 1976, 443, 613-617.

(33) Moore, D. J.; Rerek, M. E.; Mendelsohn, R. Lipid domains and orthorhombic phases in model stratum corneum: evidence from Fourier transform infrared spectroscopy studies. Biochem. Biophys. Res. Commun. 1997, 231, 797-801.

(34) Garidel, P.; Fölting, B.; Schaller, I.; Kerth, A. The microstructure of the stratum corneum lipid barrier: mid-infrared spectroscopic studies of hydrated ceramide:palmitic acid:cholesterol model systems. Biophys. Chem. 2010, 150, 144-156.

(35) Moore, D. J.; Rerek, M. E.; Mendelsohn, R. FTIR spectroscopy studies of the conformational order and phase behavior of ceramides. J. Phys. Chem. B 1997, 101, 8933-8940.

(36) Wertz, P. Epidermal lipids. In Physiology, Biochemistry and Molecular Biology of the Skin; Goldsmith, L. A., Ed. Oxford University Press: Oxford, 1991; pp. 205-235.

(37) van Smeden, J.; Boiten, W. A.; Hankemeier, T.; Rissmann, R.; Bouwstra, J. A.; Vreeken, R. J. Combined LC/MS-platform for analysis of all major stratum corneum lipids, and the profiling of skin substitutes. Biochim. Biophys. Acta 2014, 1841, 70-79. 
(38) Paz Ramos, A.; Gooris, G.; Bouwstra, J.; Molinari, M.; Lafleur, M. Raman and AFM-IR chemical imaging of stratum corneum model membranes. Can. J. Chem. 2020, 98, 495-501.

(39) Knothe, G.; Dunn, R. O. A comprehensive evaluation of the melting points of fatty acids and esters determined by differential scanning calorimetry. J. Am. Oil Chem. Soc. 2009, 86, 843-856.

(40) Janůšová, B.; Zbytovská, J.; Lorenc, P.; Vavrysová, H.; Palát, K.; Hrabálek, A.; Vávrová, K. Effect of ceramide acyl chain length on skin permeability and thermotropic phase behavior of model stratum corneum lipid membranes. Biochim. Biophys. Acta 1811, 1811, 129137.

(41) Mendelsohn, R.; Moore, D. J. Vibrational spectroscopic studies of lipid domains in biomembranes and model systems. Chem. Phys. Lipids 1998, 96, 141-157.

(42) Uchiyama, M.; Oguri, M.; Mojumdar, E. H.; Gooris, G. S.; Bouwstra, J. A. Free fatty acids chain length distribution affects the permeability of skin lipid model membranes. Biochim. Biophys. Acta 2016, 1858, 2050-2059.

(43) Moore, D. J.; Snyder, R. G.; Rerek, M. E.; Mendelsohn, R. Kinetics of membrane raft formation: fatty acid domains in stratum corneum lipid models. J. Phys. Chem. B 2006, 110, 2378-2386.

(44) Schmitt, T.; Gupta, R.; Lange, S.; Sonnenberger, S.; Dobner, B.; Hauß, T.; Rai, B.; Neubert, R. H. H. Impact of the ceramide subspecies on the nanostructure of stratum corneum lipids using neutron scattering and molecular dynamics simulations. Part I: impact of CER[NS]. Chem. Phys. Lipids 2018, 214, 58-68.

(45) Höltje, M.; Förster, T.; Brandt, B.; Engels, T.; von Rybinski, W.; Höltje, H.-D. Molecular dynamics simulations of stratum corneum lipid models: fatty acids and cholesterol. Biochim. Biophys. Acta 2001, 1511, 156-167.

(46) Wang, E.; Klauda, J. B. Molecular structure of the long periodicity phase in the stratum corneum. J. Am. Chem. Soc. 2019, 141, 16930-16943.

(47) Tang, H.-f.; Zhong, H.; Zhang, L.-l.; Gong, M.-x.; Song, S.-q.; Tian, Q. P. Theoretical investigations into the intermolecular hydrogen-bonding interactions of $\mathrm{N}$-(hydroxymethyl)acetamide dimers. J. Mol. Model. 2018, 24, 139.

(48) Del Regno, A.; Notman, R. Permeation pathways through lateral domains in model membranes of skin lipids. Phys. Chem. Chem. Phys. 2018, 20, 2162-2174.

(49) Gupta, R.; Rai, B. Molecular dynamics simulation study of skin lipids: effects of the molar ratio of individual components over a wide temperature range. J. Phys. Chem. B 2015, 119, 11643-11655.

(50) Wang, E.; Klauda, J. B. Simulations of pure ceramide and ternary lipid mixtures as simple interior stratum corneum models. $J$. Phys. Chem. B 2018, 122, 2757-2768.

(51) Smith, R.; Tanford, C. Hydrophobicity of long chain n-alkyl carboxylic acids, as measured by their distribution between heptane and aqueous solutions. Proc. Natl. Acad. Sci. U. S. A. 1973, 70, 289293.

(52) Jakobsson, A.; Westerberg, R.; Jacobsson, A. Fatty acid elongases in mammals: their regulation and roles in metabolism. Prog. Lipid Res. 2006, 45, 237-249.

(53) Sassa, T.; Ohno, Y.; Suzuki, S.; Nomura, T.; Nishioka, C.; Kashiwagi, T.; Hirayama, T.; Akiyama, M.; Taguchi, R.; Shimizu, H.; Itohara, S.; Kihara, A. Impaired epidermal permeability barrier in mice lacking elovl1, the gene responsible for very-long-chain fatty acid production. Mol. Cell. Biol. 2013, 33, 2787-2796.

(54) Školová, B.; Janǔšová, B.; Zbytovská, J.; Gooris, G.; Bouwstra, J.; Slepička, P.; Berka, P.; Roh, J.; Palát, K.; Hrabálek, A.; Vávrová, K. Ceramides in the skin lipid membranes: length matters. Langmuir 2013, 29, 15624-15633. 